\title{
Library-Based Sparse Interpolation and Super-Resolution of S/TEM Images of Biological and Material Nano-Structures
}

\author{
Suhas Sreehari ${ }^{1}$, S. V. Venkatakrishnan ${ }^{2}$, Jeff Simmons ${ }^{3}$, Lawrence Drummy ${ }^{3}$, and Charles A. Bouman ${ }^{1}$. \\ 1. Electrical and Computer Engineering, Purdue University, West Lafayette, IN, USA. \\ 2. Advanced Light Source, Lawrence Berkeley National Laboratory, Berkeley, CA, USA. \\ 3. Air Force Research Laboratory, Dayton, OH, USA.
}

Scanning transmission electron microscopes are extensively used for characterization of biological and material samples at the nano-meter scale. However, raster scanning an electron beam across a large field of view is time consuming and can damage the sample. Additionally, in order to form large field of view raster scanned images in a reasonable amount of time, during which the instrument remains at optimal stability, spatial resolution is typically compromised and a standard raster scan size is used (e.g. $2048 \times 2048$ or $4094 \times 4096$ ). This leaves significant opportunities for super-resolution and sparse interpolation image reconstruction. For these reasons, there has been a growing need to accurately reconstruct images from sparsely sampled or low-resolution S/TEM images. In many cases, images of biological and material samples contain many structures that are similar or identical to each other. Inspired by the success of modern patch-based denoising filters like non-local means (NLM) in exploiting non-local image redundancies, there have been several efforts to solve the sparse interpolation and superresolution problems using patch-based models, dictionary learning and example-based methods $[1,2]$. In any case, there is no general framework to use any generic denoising algorithm (with or without a patch library) as a prior model to perform sparse interpolation and super-resolution.

In this paper, we introduce a novel algorithm for sparse image interpolation that uses a library of high-resolution image patches to produce an interpolation with appropriate textures and edge features. In addition to reducing mean squared error in the interpolation, our library-based nonlocal means (LB-NLM) interpolation produces interpolated textures that are visually consistent with the true textures in the S/TEM images. Additionally, the interpolated images lack typical artifacts such as jaggies (see Figs. 1 and 2). The key step in our solution is to use the LB-NLM filter as a prior model in our regularized model-based interpolation. To perform this challenging step, we use the plug-and-play ( $\mathrm{P} \& \mathrm{P})$ framework [3] that is based on the alternating direction method of multipliers (ADMM) [4] and decouples the forward model and the prior model terms in the maximum $a$ posteriori (MAP) cost function. This results in an algorithm that involves repeated application of two steps: an inversion step only dependent on the forward model, and a denoising step only dependent on the image prior model. The P\&P algorithm takes ADMM a step further by replacing the prior model minimization by a denoising operator. Furthermore, the $\mathrm{P} \& \mathrm{P}$ algorithm is shown to converge if the denoising operator has a doubly stochastic gradient [3]. Since LB-NLM weights only the patches from the external library, the gradient of LB-NLM is a diagonal matrix, making it trivially doubly stochastic. Our results demonstrate that using LB-NLM as a prior model within the powerful P\&P framework can improve image interpolation quality well beyond linear methods. 


\section{References:}

[1] C. Guillemot and O. Le Meur, "Image inpainting: Overview and recent advances," Signal Processing Magazine, IEEE, 31: 1, pp. 127-144, Jan 2014.

[2] S. C. Park, M. K. Park, and M. G. Kang, "Super-resolution image reconstruction: a technical overview," Signal Processing Magazine, IEEE, 20:3, pp. 21-36, 2003.

[3] S. Sreehari, S. Venkatakrishnan, B. Wohlberg, L. F. Drummy, J. P. Simmons, and C. A. Bouman, "Plug-and-play priors for bright field electron tomography and sparse interpolation," arXiv preprint arXiv:1512.07331, 2015.

[4] D. Gabay and B. Mercier, "A dual algorithm for the solution of nonlinear variational problems via finite element approxi- mation," Computers \& Mathematics with Applications, 2:1, pp. 17-40, 1976.

[5] This work was supported by AFOSR/MURI grant \#FA9550-12-1-0458, by UES Inc.

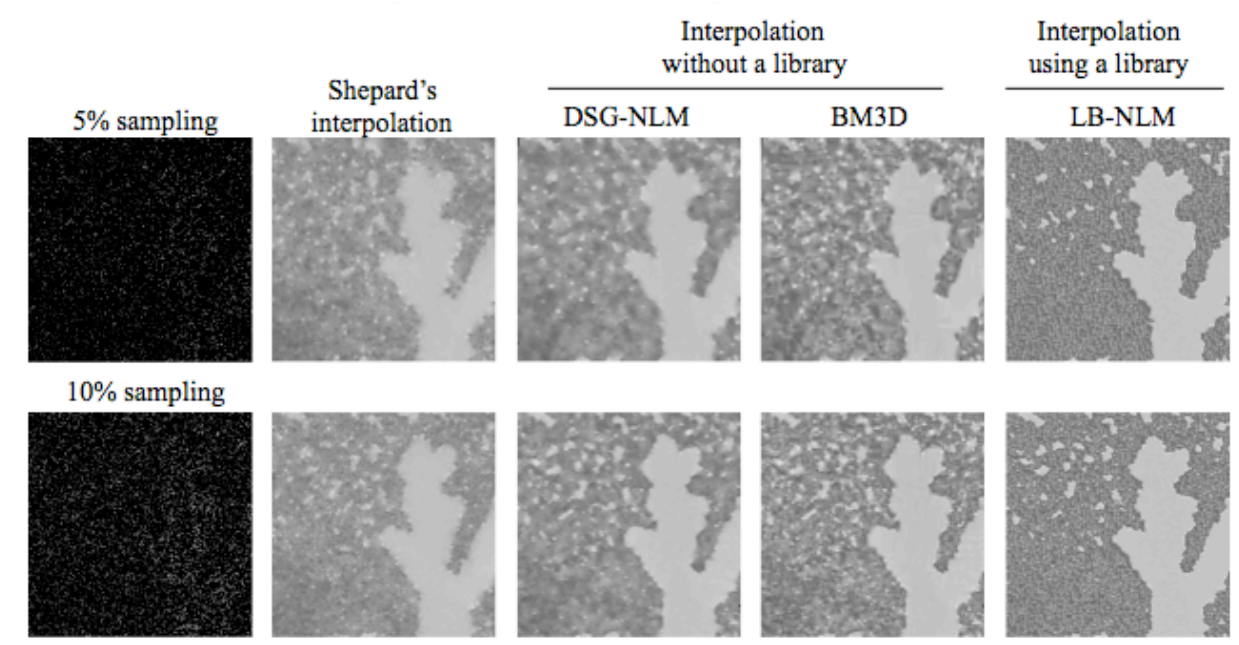

Figure 1. Interpolation of a $256 \times 256$ TEM image of iridovirus assemblies.

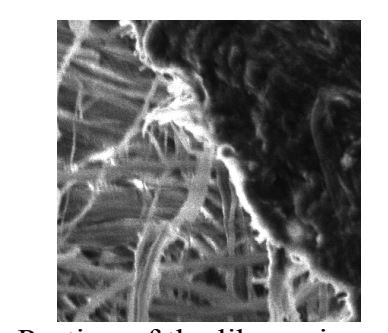

Portion of the library image

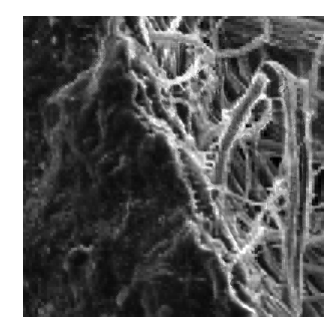

DSG-NLM interpolation

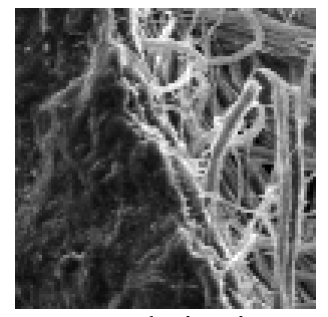

Low resolution image

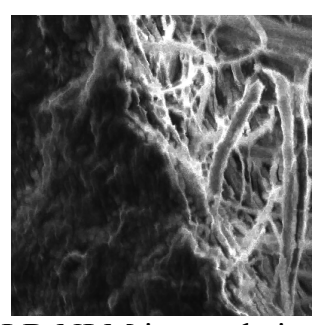

LB-NLM interpolation

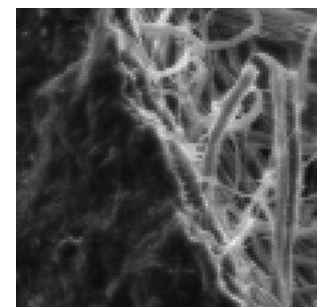

Shepard's interpolation

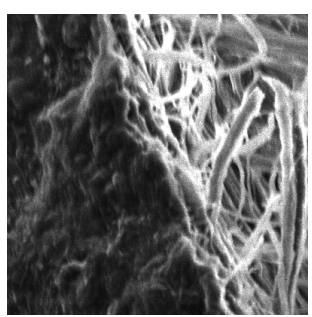

Ground truth

Figure 2. $4 x$ super-resolution of a $100 \times 100$ SEM image of surface crack in the shell of the marine mollusk Hinea brasiliana. 